\section{CLINICAL REPORT}

A. Yamamoto

Y. Kikuchi

K. Homma

T. O’uchi

S. Furui

\title{
Characteristics of Intravascular Large B-Cell Lymphoma on Cerebral MR Imaging
}

\begin{abstract}
SUMMARY: IVL is characterized by a propensity for intravascular tumor cell proliferation. Premortem diagnosis of IVL is difficult because of its nonspecific clinical, laboratory, and imaging manifestations. This study examined cerebral MR imaging patterns of IVL and their changes with and without chemotherapy. Nine of 11 patients studied presented with abnormal findings. We define 5 patterns of abnormal MR imaging findings: 1) infarctlike lesions, 2) nonspecific white matter lesions, 3) meningeal enhancement, 4) masslike lesions, and 5) hyperintense lesions in the pons on T2WI. Seven patients presented with only 1 pattern, while 2 patients presented with multiple patterns. Lesions in 7 treated patients responded to chemotherapy. Pathologic specimens revealed intravascular tumor cell infiltration with associated infarctions, necrosis, congestion, demyelination, vasculitis, and tumor cell extravasation. We conclude that MR imaging patterns can be possible manifestations of intravasculardominant infiltration by tumor cells with associated occlusion or inflammation, depending on the level of affected vessels.
\end{abstract}

ABBREVIATIONS: IVL = intravascular large B-cell lymphoma; PRES $=$ posterior reversible encephalopathy syndrome; R-CHOP = rituximab with cyclophosphamide, vincristine, doxorubicin, and prednisolone

$l^{N}$ VL is a rare subtype of extranodal diffuse large B-cell lymphoma. ${ }^{1}$ It is characterized by a propensity for intravascular proliferation of tumor cells in small vessels with a predilection for the central nervous system and skin. ${ }^{2,3}$ Premortem diagnosis of IVL is difficult because of its variable clinical manifestations and nonspecific laboratory findings, though the elevation of $\mathrm{LDH}$ and the soluble interleukin-2 receptor is found in many cases. ${ }^{4-6}$ Cerebral MR imaging findings in patients with IVL are also diverse; these variations make diagnosis challenging. ${ }^{7}$ Once proper diagnosis of IVL is determined by tissue biopsy, complete remission can be achieved by chemotherapy by using R-CHOP. ${ }^{8,9}$ Recognition and careful interpretation of the various findings on cerebral MR imaging may facilitate early diagnosis and intervention and improve the prognosis of this often-missed disease. ${ }^{10}$

\section{Case Series}

\section{Subjects and Methods}

The Institutional Committee for Medical Research Ethics approved this retrospective study and waived informed consent. We retrospectively reviewed cerebral MR imaging of 11 consecutive patients who were diagnosed pathologically with IVL between 1998 and 2009. Two patients were diagnosed at postmortem examination, and 9 cases were diagnosed from various tissue biopsies. The biopsies included 1 lymph node, 1 lung and bone marrow, and 7 random skin biopsies. Three of 9 patients who were diagnosed by tissue biopsy underwent

Received March 1, 2011; accepted after revision May 11.

From the Department of Radiology (A.Y., S.F.), Teikyo University School of Medicine, Tokyo, Japan; Department of Radiology (Y.K., T.O.), Kameda Medical Center, Chiba, Japan; and Department of Pathology (K.H.), Dokkyo University School of Medicine, Tochigi, Japan.

Please address correspondence to Asako Yamamoto, MD, Department of Radiology, Teikyo University School of Medicine, Tokyo, Japan, 2-11-1, Kaga, Itabashiku, Tokyo, Japan; e-mail: asakonemurinomori@yahoo.co.jp

EIndicates article with supplemental on-line table.

http://dx.doi.org/10.3174/ajnr.A2770 autopsy. Medical charts were reviewed for their symptoms, past history, laboratory data, and treatment.

MR imaging was performed on a $1.5 \mathrm{~T}$ unit (Magnetom Vision; Siemens Healthcare, Erlangen, Germany). Axial T2weighted fast spin-echo imaging $(\mathrm{TR}=4000 \mathrm{~ms}, \mathrm{TE}=99 \mathrm{~ms})$, coronal T1-weighted imaging $(\mathrm{TR}=570 \mathrm{~ms}, \mathrm{TE}=12 \mathrm{~ms})$, axial fluid-attenuated inversion recovery imaging $(\mathrm{TR}=9000$ $\mathrm{ms}, \mathrm{TE}=110 \mathrm{~ms}$, TI $=2200 \mathrm{~ms}), \mathrm{DWI}(b=1000, \mathrm{TR}=2200$ $\mathrm{ms}, \mathrm{TE}=103.0 \mathrm{~ms}$ ), and axial, coronal and sagittal MR images after intravenous administration of $0.1-\mathrm{mmol} / \mathrm{kg}$ gadopentetate dimeglumine were available for all patients. Seven of 11 patients underwent initial MR imaging within 1 week from admission, and the other 4 were scanned within 2 months.

Three radiologists reviewed the cerebral MR imaging, and findings were categorized as follows: 1) infarctlike lesions, 2) nonspecific white matter lesions, 3) meningeal enhancement, 4) masslike lesions, or 5) hyperintense lesions in the pons on T2WI. The criterion for infarctlike lesions was hyperintense areas on T2WI with diffusion restriction. Nonspecific white matter lesions were defined as poorly margined hyperintense lesions on T2WI without mass effect or abnormal enhancement. The criterion for meningeal enhancement was abnormal enhancement along the surface of the cortex with a piaarachnoid pattern extending $>1$ gyrus in $>2$ planes on postcontrast T1-weighted images. The criterion used for masslike lesions was intraparenchymal focal areas with contrast enhancement.

\section{Results}

The patients' characteristics, symptoms, primary cerebral MR imaging findings, and changes in findings on follow-up MR imaging studies are summarized in On-line Table. The patients were 4 men and 7 women, ranging from 63 to 84 years of age (average, 71.4 years of age). Presenting symptoms were fever in all, dementia in 5 , shortness of breath in 2 , personality changes in 2, and malaise in 1 patient. Five patients, including 3 treated and 2 untreated, underwent brain postmortem examination (Table). 


\begin{tabular}{|c|c|c|c|c|c|}
\hline \multicolumn{6}{|c|}{ Findings on brain postmortem examination } \\
\hline Case & $\begin{array}{l}\text { Tumoral Vascular } \\
\text { Occlusion }\end{array}$ & Infarction & Demyelination & Meningitis & $\begin{array}{c}\text { Tumor Cell } \\
\text { Extravasation }\end{array}$ \\
\hline 1 & $x$ & $\begin{array}{l}\mathrm{X} / \text { multiple hemorrhagic infarcts with } \\
\text { necrosis and surrounding congestion }\end{array}$ & X/cerebral WM & & \\
\hline 2 & X (including pons) WM & $\begin{array}{l}\mathrm{X} / \text { multiple hemorrhagic infarcts, } \\
\text { thickening and subendothelial tumoral } \\
\text { infiltration }\end{array}$ & $\mathrm{X} /$ cerebral/cerebellar & $\mathrm{X} /$ vascular wall & \\
\hline 3 & $X$ (including pons) & $\begin{array}{l}\mathrm{X} / \text { multiple hemorrhagic infarcts with } \\
\text { necrosis and surrounding congestion }\end{array}$ & $\mathrm{X} /$ cerebral WM & & $x$ \\
\hline 5 & $x$ & $\mathrm{X} /$ multiple infarcts & X/cerebral WM & $\begin{array}{l}\text { (Not applicable to } \\
\text { the corresponding } \\
\text { area) }\end{array}$ & \\
\hline 10 & $\begin{array}{l}\text { No tumor cell infiltration } \\
\text { detected }\end{array}$ & & & & \\
\hline
\end{tabular}

Note:- $\mathrm{X}$ indicates presence of finding.
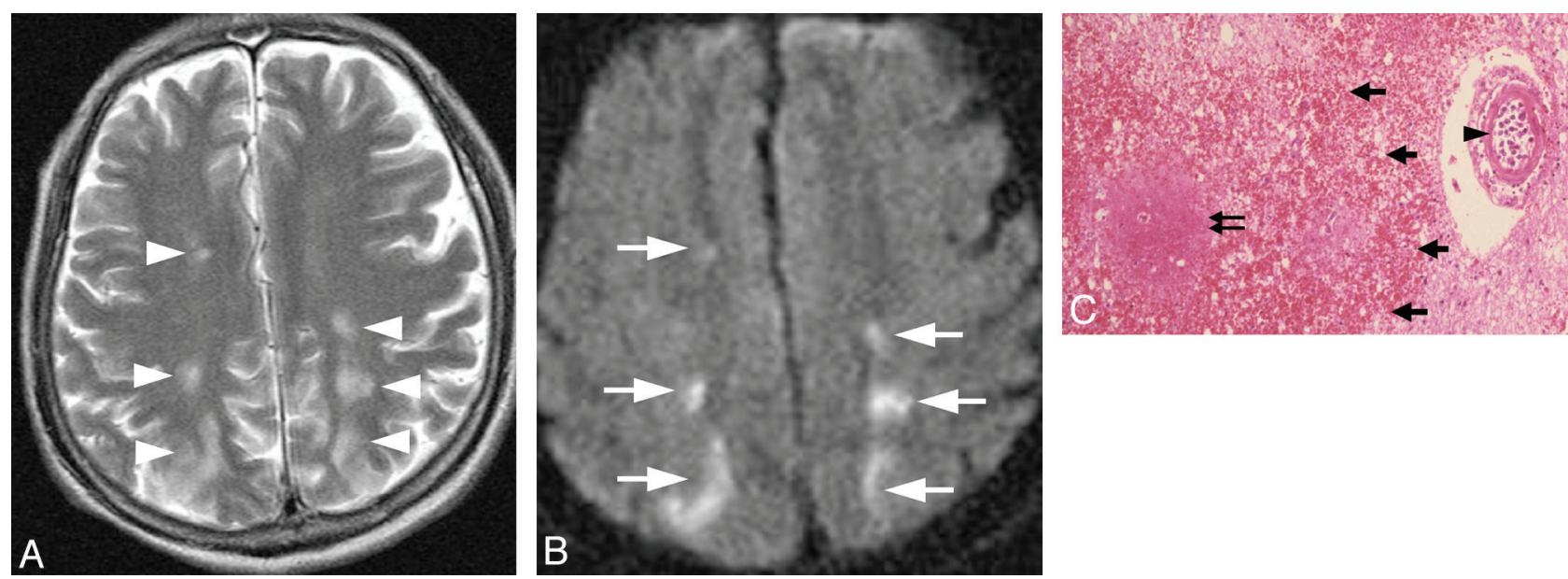

Fig 1. Case 1. Infarctlike lesion in a 74-year-old man. $A$, Axial T2WI shows hyperintense spots in the bilateral watershed area (white arrowheads). $B$, The corresponding areas show diffusion restriction on DWI (white arrows). C, Pathologic specimen shows irregular-shaped necrosis (double black arrow) and congestion (thick black arrows) surrounding the occluded vessel (arrowhead) (hematoxylin-eosin, original magnification $\times 25$ ).

Cerebral MR imaging was performed on 11 patients before treatment and showed abnormal findings in 9 patients. Seven patients presented with a single type of lesion, and 2 patients had multiple types of lesion. Two patients were not diagnosed until postmortem examination, so they were untreated for IVL. The remaining 7 patients with abnormal findings were treated for IVL, and all their lesions responded to chemotherapy. The lesions worsened in the 2 untreated patients.

Infarctlike lesions were observed in 2 of 11 patients, appearing as multiple hyperintense spots on T2WI with diffusion restriction in white matter, including the periventricular areas, watershed territory, and corpus callosum (Fig 1A, -B). In these patients, the multifocal lesions decreased in size and number following treatment. Postmortem examination revealed multiple infarctions with vascular occlusion by tumor cells, irregular-shaped necrosis, and congestion around the occluded vessels (Fig 1C). Minor hemorrhagic infarctions were also frequently observed. Nonspecific white matter lesions were observed in 2 of the 11 patients. Diffuse white matter hyperintensity was seen especially in the periventricular areas (Fig $2 A$ ). Focal white matter hyperintensity was also seen in subcortical areas in case 3. In this patient who was not diagnosed and treated specifically for IVL, the hyperintense area gradually increased in size. However in the treated patient, the lesions improved promptly after treatment started (Fig $2 B$ ).
Postmortem examination revealed diffuse demyelination of white matter with vessels severely occluded by tumor cells in the corresponding areas (Fig 2C).

Meningeal enhancement was observed in 2 patients (Fig $3 A$ ). In 1 of 2 patients with meningeal enhancement, the enhancement decreased just after treatment started. No surrounding edema or intraparenchymal enhanced lesions were observed. Postmortem examination revealed thickened vascular walls and necrotic and subendothelial tumor infiltration with abundant lymphocytes surrounding the vascular walls (Fig 3B). Masslike lesions were observed in 1 patient with multiple intraparenchymal focal enhanced lesions and mass effect (Fig 4A). No abnormal findings were found on DWI (Fig 4B). A gradual decrease in the contrast enhancement and mass effect of the lesions was observed after chemotherapy was started (Fig $4 C$ ). No postmortem examination was performed on this patient. The hyperintense lesions in the pons on T2WI without diffusion restriction or contrast enhancement were observed in 5 of 11 patients. This signal-intensity pattern was the only abnormal one in 4 of the 5 patients. All 5 patients showed symmetric hyperintense areas in the central pons, sparing the pontine tegmentum and ventrolateral pons (Fig $5 A$ ). The hyperintense areas in the pons decreased in size and intensity in all of the 4 patients treated (Fig $5 B$ ). In case 9 , a follow-up MR imaging showed a decrease in the size of the lesion 3 days after 

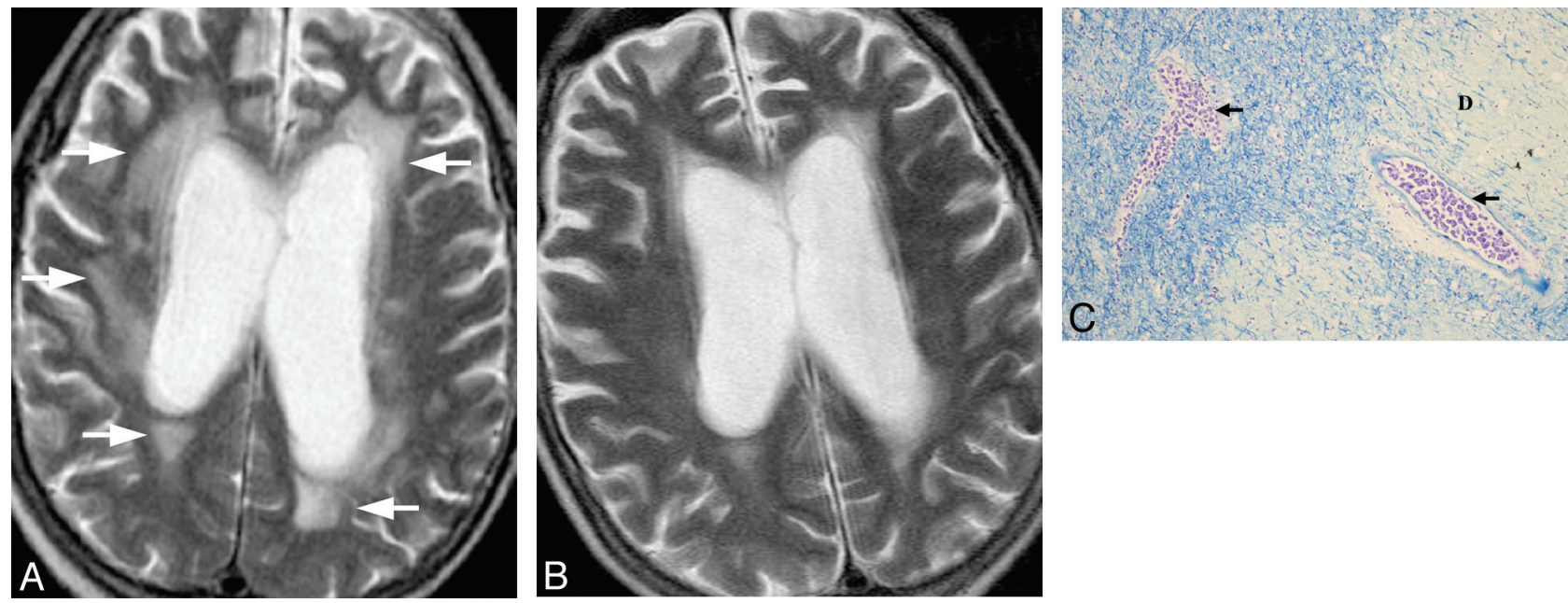

Fig 2. Case 2. Nonspecific white matter lesion in a 69-year-old man. $A$, Axial T2WI shows diffuse hyperintensity in the bilateral periventricular areas before treatment (white arrows). $B$, Posttreatment T2WI on day 94 shows a decrease in the abnormal signal intensity. $C$, Pathologic specimen shows occlusion of the vessels by tumor cells (black arrows) and diffuse demyelination (D) with or without evidence of infarction (Klüver-Barrera, original magnification $\times 25$ ).
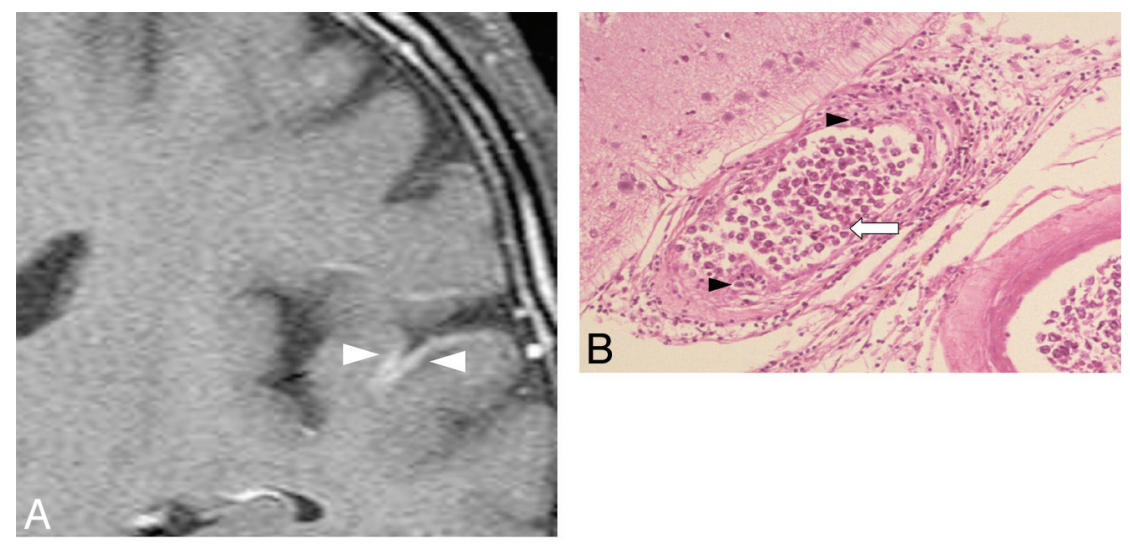

Fig 3. Case 2. Meningeal enhancement in a 69-year-old man. $A$, Gadolinium-enhanced coronal T1-weighted image shows abnormal meningeal enhancement around the temporal lobe before treatment (white arrowheads). B, Pathologic specimen shows thickening of the affected vessel walls with intraluminal (white arrow) and subendothelial (black arrowheads) tumoral infiltration (hematoxylin-eosin, original magnification $\times 25$ ).

treatment. Brain stem atrophy was not observed in any of these 4 cases after treatment.

Of all 11 patients, no patient had inappropriate fluid intake or other conditions that may predispose to osmotic demyelination syndrome. The patients did not present with severe hypertension, seizure, reaction to chemotherapy, or other conditions that may lead to PRES.

\section{Discussion}

We documented brain MR imaging findings from 11 patients with IVL and defined 5 patterns of abnormal findings; 1 ) infarctlike lesions, 2) nonspecific white matter lesions, 3) meningeal enhancement, 4) masslike lesions, and 5) hyperintense lesions in the pons on T2WI.

\section{Infarctlike Lesions}

Infarctlike lesions suggest that the tumor predominantly involves small arteries. ${ }^{11}$ This pattern was reported in $36 \%$ of the patients with IVL who had brain MR imaging. ${ }^{7}$ Although infarctlike lesions were observed in only 2 patients in our study (2/11), 4 of the 5 postmortem examinations, including those of 2 patients without infarctlike lesions on MR imaging, re- vealed multiple infarctions and severe vascular occlusions by tumor cells. The low frequency of infarctlike lesions may be explained by the timing of pretreatment MR imaging and the stage of the disease.

\section{Nonspecific White Matter Lesions}

Poorly defined nonspecific white matter lesions have been reported, especially in the periventricular area, and our study showed a similar distribution. ${ }^{7,12}$ In the 2 patients with this pattern, the signal intensity decreased in 1 patient after treatment started and increased in the other patient who went without treatment for IVL. Postmortem examination revealed leukoencephalopathy and severe tumor cell infiltration. The small vessels severely occluded by tumor cells observed at postmortem examination were much more frequent than the number of infarct foci on MR imaging. Usually the tumor cells are noncohesive and free in the lumina in IVL, but the pathogenesis of this pattern has been described as sluggish flow within the lumens of capillaries or microinfarcts. ${ }^{12}$ We speculate this pattern is caused by congestion and chronic ischemic change due to severe vascular infiltration by tumor cells. 

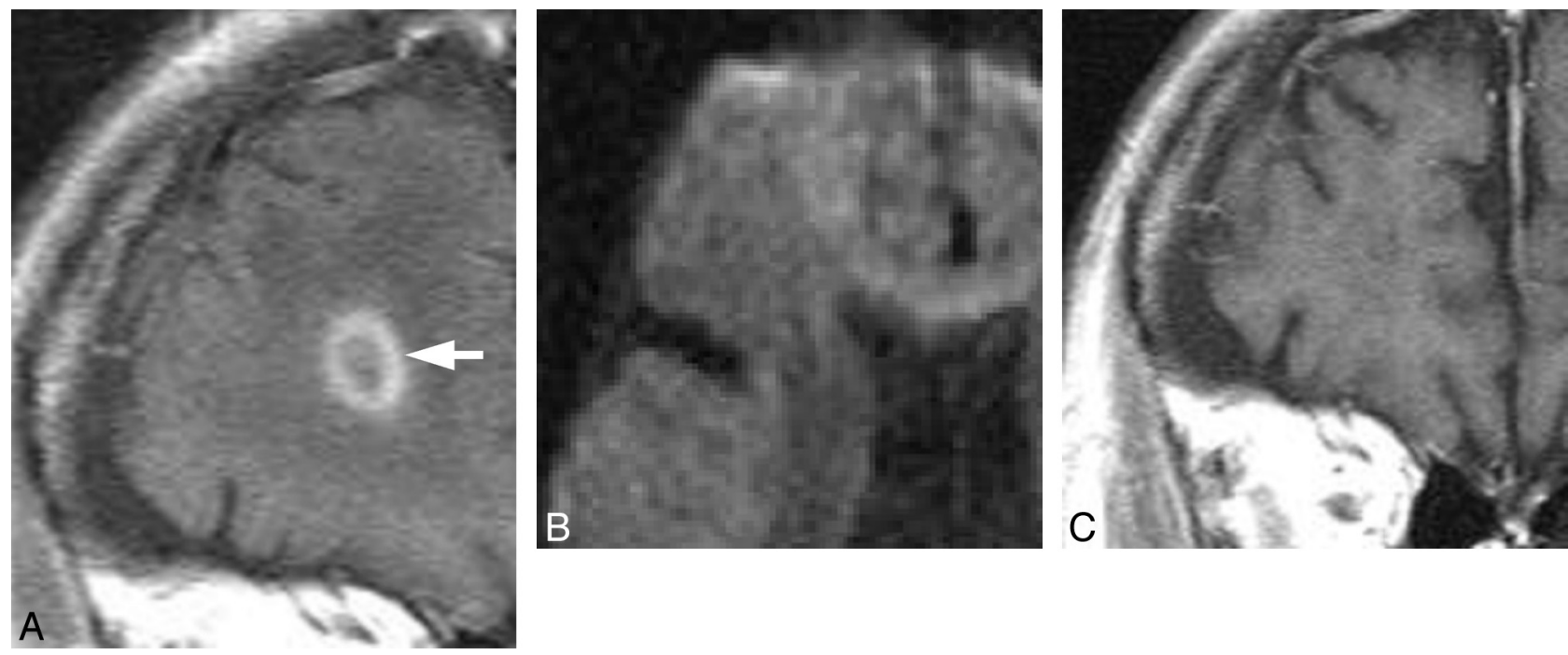

Fig 4. Case 4. Masslike lesion in a 70-year-old man. $A$, Coronal gadolinium-enhanced T1-weighted image shows ringlike enhancement before treatment (white arrow). $B$, Axial DWI shows no abnormal signal intensity in the lesion. $C$, Follow-up gadolinium-enhanced coronal T1-weighted image shows regression of the enhancement after treatment on day 121 .
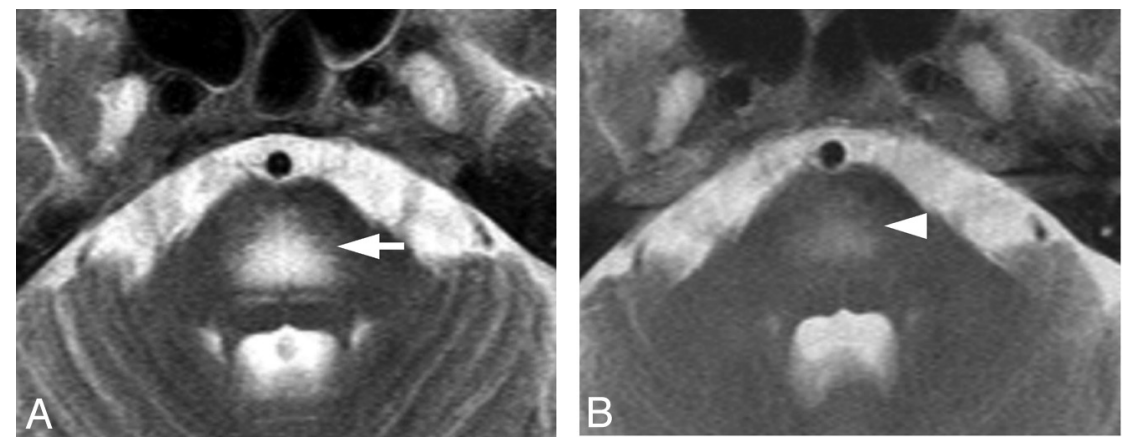

Fig 5. Case 8. Hyperintense lesion in the pons on a T2-weighted image in a 63-year-old woman. $A$, Axial T2WI shows symmetric hyperintense lesions in the center of the pons sparing the pontine tegmentum and ventrolateral pons (white arrow). DWI showed T2 shinethrough without diffusion restriction (not shown). $B$, T2WI after chemotherapy shows a decreased abnormal signal intensity in the pons on day 85 (white arrowhead).

\section{Meningeal Enhancement}

Meningeal enhancement is sometimes present in patients with IVL, though the nature of the finding has not been determined. ${ }^{7}$ Thrombus formation, vasculitis with lymphocyte infiltration, and fibrous thickening of the outer layer of small vessels have been reported in the cerebral vessels at postmortem examination. ${ }^{2,13}$ Postmortem examination of cases in this study showed a severe meningeal inflammatory reaction with tumor cells. This pathologic finding suggests the invasion pathway of lymphoma cells to the extravascular structure.

\section{Masslike Lesions}

Intraparenchymal masslike lesions presented with extensive vasogenic edema and mass effect, contrary to the characteristic features of tumor cell infiltration predominantly in the vascular lumen. The response to therapy strongly suggested that the lesions were caused by IVL. Masslike lesions have been described in previous reports as representing extravascular spread of lymphoma cells with accompanying inflammatory change in vascular walls and surrounding parenchyma with microinfarct. ${ }^{2,3,7,14,15}$ Extravasation of lymphoma cells and vascular wall thickening with direct infiltration of tumor cells into the vascular walls were present at postmortem examina- tion. The pathologic change behind this pattern may be multifactorial.

\section{Hyperintense Lesions in the Pons on T2WI}

Hyperintense lesions in the central pons on T2WI without enhancement or diffusion restriction were observed in 5 of 11 patients with IVL before treatment. This pattern in patients with IVL has not previously been reported in English-language literature, to our knowledge. The decrease of the lesions seen on MR imaging in 4 patients after treatment started strongly suggests that these lesions are a manifestation of IVL. The hyperintense lesions in the central pons, excluding the pontine tegmentum and ventrolateral region, on T2WI are similar to findings in pontine osmolytic demyelination syndrome and PRES in the brain stem or intracranial dural arteriovenous fistula with venous congestion. ${ }^{16-20}$ Five patients showing hyperintense lesions in the central pons on T2WI had no indication of osmolytic demyelination syndrome or typical PRES. Although there was no correlating pathologic evidence from the patients who presented with this pattern, 2 of the 5 postmortem examinations on other patients showed tumor cell infiltration into the vessels including the pons (Fig 6). We speculate that vascular occlusion in small veins and arteries by 


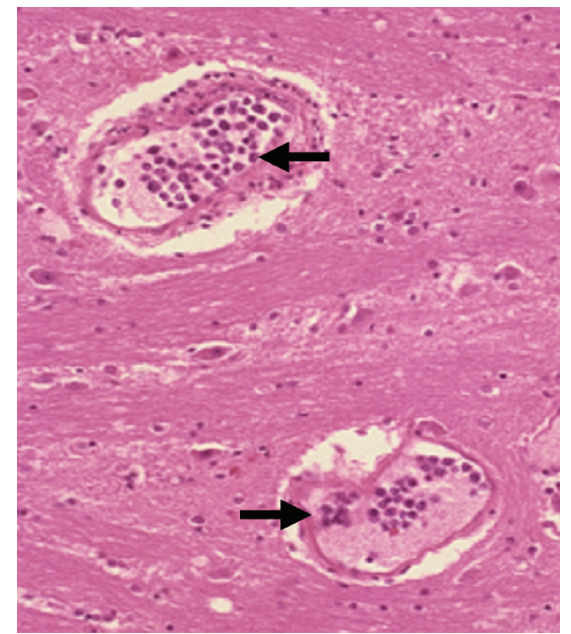

Fig 6. Case 3. Tumor cell infiltration into the pontine vessels in an 84-year-old man. Pathologic specimen shows lymphoma cell infiltration into the capillaries in the pons (black arrows) (hematoxylin-eosin, original magnification $\times 50$ ).

tumor cells results in venous congestion that could manifest as hyperintensity in the central pons on T2WI.

Cerebral MR imaging findings in patients with IVL may be diverse, but when considered in conjunction with pathologic findings, the various MR imaging findings can be logically interpreted. When occlusions occur in small arteries, one can expect multiple small infarctions such as the infarctlike lesions seen on MR imaging in this study. Occlusion in capillaries would cause diffuse white matter abnormal intensities. We speculated that the hyperintense lesions in the pons were also a result of occlusion of capillaries in the pons as was observed at postmortem examination in case 3 . When large numbers of lymphoma cells cause extravascular mass, masslike lesions can be expected. Thickening of the meningeal vessels with some inflammatory reaction would present as meningeal enhancement. Attention to the intravascular nature of this unique lymphoma could lead to better recognition of the various MR imaging appearances and a more timely diagnosis.

This study has several limitations. The number of patients is small due to the rarity of this tumor, and radiologic follow-up is short. Cerebral postmortem examination was performed in 5 cases, including 1 case in which only the bilateral frontal lobes were examined. Brain tissue was not taken from the remaining 6 cases. Masslike lesions and hyperintense lesions in the pons on T2WI were not pathologically proved in this study. Brain biopsy is often difficult in patients with severe conditions, and postmortem examination might be the only way to analyze these patterns.

\section{Conclusions}

Infarctlike lesions, nonspecific white matter lesions, meningeal enhancement, masslike lesions, and the hyperintense lesions in the pons on T2WI were observed on MR imaging in 9 patients with IVL. Each of the lesions described responded to proper chemotherapy. It is important to recognize these MR imaging patterns as possible manifestations of intravasculardominant infiltration by tumor cells with varying degrees of associated occlusion or inflammation, depending on the level of the affected vessels. Prompt recognition of these imaging patterns may lead to early diagnosis of IVL with improved prognosis.

\section{Acknowledgments}

We thank Desmond Bell for his assistance.

\section{References}

1. Nakamura S, Ponzoni M, Campo E. Intravascular large-B-cell lymphoma. In: Swerdlow SH, Campo E, Harris NL, et al, eds. World Health Organization: Pathology and Genetics of Tumors of Haematopoietic and Lymphoid Tissues. 4th ed. Lyon, France: IARC Press; 2008:252-53

2. Wick MR, Mills SE. Intravascular lymphomatosis: clinicopathologic features and differential diagnosis. Semin Diagn Pathol 1991;8:91-101

3. Glass J, Hochberg FH, Miller DC. Intravascular lymphomatosis: a systemic disease with neurologic manifestations. Cancer 1993;71:3156-64

4. Shimada K, Kinoshita T, Naoe T, et al. Presentation and management of intravascular large B-cell lymphoma. Lancet Oncol 2009;10:895-902

5. Ferreri AJ, Campo E, Seymour JF, et al. Intravascular lymphoma: clinical presentation, natural history, management and prognostic factors in a series of 38 cases, with special emphasis on the "cutaneous variant." Br J Haematol 2004;127:173-83

6. Asada N, Odawara J, Kimura S, et al. Use of random skin biopsy for diagnosis of intravascular large B-cell lymphoma. Mayo Clin Proc 2007;82:1525-27

7. Williams RL, Meltzer CC, Smirniotopoulos JG, et al. Cerebral MR imaging in intravascular lymphomatosis. AJNR Am J Neuroradiol 1998;19:427-31

8. Shimada K, Matsue K, Yamamoto K, et al. Retrospective analysis of intravascular large B-cell lymphoma treated with rituximab-containing chemotherapy as reported by the IVL study group in Japan. J Clin Oncol 2008;26:3189-95

9. Han K, Haley JC, Carlson K, et al. Regression of cutaneous intravascular lymphoma with rituximab. Cutis 2003;72:137-40

10. Domizio P, Hall PA, Cotter F, et al. Angiotropic large cell lymphoma (ALCL): morphological, immunohistochemical and genotypic studies with analysis of previous reports. Hematol Oncol 1989;7:195-206

11. Ganguly S. Acute intracerebral hemorrhage in intravascular lymphoma: a serious infusion related adverse event of rituximab. Am J Clin Oncol 2007;30:211-12

12. Liew CL, Shyu WC, Tsao WL, et al. Intravascular lymphomatosis mimicks a cerebral demyelinating disorder. Acta Neurol Taiwan 2006;15:264-68

13. Amagasaki K, Yamazaki H, Ohmori K, et al. Malignant intravascular lymphomatosis associated with venous stenosis: case report. J Neurosurg 1999; 90:355-58

14. Wick MR, Mills SE, Scheithauer BW, et al. Reassessment of malignant "angioendotheliomatosis": evidence in favor of its reclassification as "intravascular lymphomatosis." Am J Surg Pathol 1986;10:112-23

15. Fredericks RK, Walker FO, Elster A, et al. Angiotrophic intravascular large-cel lymphoma (malignant angioendotheliomatosis): report of a case and review of the literature. Surg Neurol 1991;35:218-23

16. Endo Y, Oda M, Hara M. Central pontine myelinolysis: a study of 37 cases in 1,000 consecutive autopsies. Acta Neuropathol 1981;53:145-53

17. Crosley CJ, Rorke LB, Evans A, et al. Central nervous system lesions in childhood leukemia. Neurology 1978;28:678-85

18. Marchioli CC, Graziano SL. Paraneoplastic syndromes associated with small cell lung cancer. Chest Surg Clin N Am 1997;7:65-80

19. Miller GM, Baker HL Jr, Okazaki H, et al. Central pontine myelinolysis and its imitators: MR findings. Radiology 1988;168:795-802

20. Iwasaki M, Murakami K, Tomita T, et al. Cavernous sinus dural arteriovenous fistula complicated by pontine venous congestion: a case report. Surg Neurol 2006;65:516-18 